\title{
Right Hemisphere Dominance during Spatial Selective Attention and Target Detection Occurs Outside the Dorsal Frontoparietal Network
}

\author{
Gordon L. Shulman, ${ }^{1}$ Daniel L. W. Pope, ${ }^{2}$ Serguei V. Astafiev, ${ }^{2}$ Mark P. McAvoy, ${ }^{2}$ Abraham Z. Snyder, ${ }^{1,2}$ \\ and Maurizio Corbetta ${ }^{1,2,3}$ \\ Departments of ${ }^{1}$ Neurology, ${ }^{2}$ Radiology, and ${ }^{3}$ Anatomy and Neurobiology, Washington University, St. Louis, Missouri 63110
}

\begin{abstract}
Spatial selective attention is widely considered to be right hemisphere dominant. Previous functional magnetic resonance imaging studies, however, have reported bilateral blood-oxygenation-level-dependent responses in dorsal frontoparietal regions during anticipatory shifts of attention to a location (Kastner et al., 1999; Corbetta et al., 2000; Hopfinger et al., 2000). Right-lateralized activity has mainly been reported in ventral frontoparietal regions for shifts of attention to an unattended target stimulus (Arrington et al., 2000; Corbetta et al., 2000). However, clear conclusions cannot be drawn from these studies because hemispheric asymmetries were not assessed using direct voxelwise comparisons of activity in left and right hemispheres. Here, we used this technique to measure hemispheric asymmetries during shifts of spatial attention evoked by a peripheral cue stimulus and during target detection at the cued location. Stimulus-driven shifts of spatial attention in both visual fields evoked right-hemisphere dominant activity in temporoparietal junction (TPJ). Target detection at the attended location produced a more widespread right hemisphere dominance in frontal, parietal, and temporal cortex, including the TPJ region asymmetrically activated during shifts of spatial attention. However, hemispheric asymmetries were not observed during either shifts of attention or target detection in the dorsal frontoparietal regions (anterior precuneus, medial intraparietal sulcus, frontal eye fields) that showed the most robust activations for shifts of attention. Therefore, right hemisphere dominance during stimulus-driven shifts of spatial attention and target detection reflects asymmetries in cortical regions that are largely distinct from the dorsal frontoparietal network involved in the control of selective attention.
\end{abstract}

\section{Introduction}

Spatial selective attention is widely considered to be right hemisphere dominant. The most cited evidence comes from studies of stroke patients with spatial neglect, a failure to perceive and respond to stimuli on the contralesional side of space, which is more severe and prolonged following right than left hemisphere lesions (Heilman et al., 1985; Bowen et al., 1999; Ringman et al., 2004; Becker and Karnath, 2007). One prominent theory of this asymmetry is that the left hemisphere controls shifts of attention in the rightward direction, while the right hemisphere controls shifts of attention in either direction (Mesulam, 1981). Several neuroimaging studies in healthy adults have reported evidence of right hemisphere dominance in tasks involving a cued shift of spatial attention followed by target detection (Nobre et al., 1997; Gitelman et al., 1999). However, later studies that separated the activations for endogenous shifts of attention from the activations to subsequent target stimuli reported bilateral activations in dorsal frontoparietal regions that are likely the primary control-

Received Aug. 18, 2009; revised Nov. 17, 2009; accepted Jan. 8, 2010

This work was supported by the National Institute of Neurological Disorders and Stroke Grant R01 NS048013, National Institute of Mental Health Grant R01 MH 71920-06, and the J. S. McDonnell Foundation.

Correspondence should be addressed to Gordon Shulman, Department of Neurology, Box 8111, 4525 Scott Avenue, Room 2109, St. Louis, M0 63110. E-mail: gordon@npg.wustl.edu.

D0I:10.1523/JNEUROSCI.4085-09.2010

Copyright $\odot 2010$ the authors $\quad 0270-6474 / 10 / 303640-12 \$ 15.00 / 0$ lers of spatial attention (Kastner et al., 1999; Corbetta et al., 2000; Hopfinger et al., 2000; Woldorff et al., 2004), sometimes with a contralateral advantage (Sylvester et al., 2007). Moreover, mapping studies have reported polar-angle representations of the contralateral field in dorsal frontoparietal regions of both hemispheres (Sereno et al., 2001; Silver et al., 2005; Hagler and Sereno, 2006; Jack et al., 2007; Swisher et al., 2007). Therefore, blood oxygenation level-dependent (BOLD) activation studies have not generally supported the proposal that the right hemisphere is dominant for the endogenous control of spatial selective attention.

Stimulus-driven shifts of spatial attention, which are evoked by unattended but behaviorally important stimuli, activate not only the bilateral dorsal network involved in endogenous spatial attention, but also a ventral network comprising right temporoparietal junction (TPJ) and right ventral frontal cortex (Arrington et al., 2000; Corbetta et al., 2000; Macaluso et al., 2002). Therefore, activation studies suggest that one of the two brain networks activated by shifts of spatial attention may be right hemisphere dominant, but is only recruited under stimulus-driven conditions.

Importantly, conclusions about hemispheric asymmetries drawn from neuroimaging studies have been criticized (Liégeois et al., 2002; Stevens et al., 2005; Macaluso and Patria, 2007) since they have not been based on direct statistical comparisons of the magnitude of activations in the two hemispheres. For example, lateralization cannot be inferred if there is a significant activation in only one hemisphere, since this result could be an artifact of statisti- 
cal thresholding. Here we tested for hemispheric asymmetries in dorsal and ventral frontoparietal regions during stimulus-driven shifts of spatial attention using direct voxelwise comparisons of left and right hemisphere activity.

Regions of both dorsal and ventral frontoparietal networks are also activated during target detection (Corbetta et al., 2000; Hopfinger et al., 2000; Hampshire et al., 2007) and right hemispheric dominant BOLD responses to auditory oddball targets have been reported in frontal, parietal, and temporal cortex (Stevens et al., 2005). We determined whether hemispheric asymmetries during target detection localized to dorsal and ventral frontoparietal networks, independently defined from their activation by stimulus-driven shifts of spatial attention, or were mainly observed outside those networks.

\section{Materials and Methods \\ Paradigm}

Subjects searched for a target object in two task-relevant rapid-serialvisual-presentation (RSVP) streams, one left and one right of fixation (Fig. 1). Each stream was flanked by three irrelevant distracter RSVP streams to increase the need for careful spatial selection. A salient, easily detectable cue stimulus (a filled red square) presented without accompanying objects in one of the task-relevant RSVP streams indicated which of the two streams contained the target. The cue was presented multiple times over a block. A cue might occur in the same stream as the previous cue, indicating that subjects should continue to monitor the same stream (a "stay" cue) or it might occur in the opposite, unattended stream, indicating that subjects should shift their attention to the new stream (a "shift" cue). Target identification was set at difficult levels (see below). The high difficulty of the target discrimination and the presence of distracter streams, coupled with the high salience of the cue stimulus, encouraged subjects to attend closely to the cued stream rather than distributing attention across both streams. In one set of scans, a cue was highly likely to occur in the same stream as the previous cue (i.e., the probability that the cue was a stay cue was 0.86 ); in another set of scans, a cue was highly likely to occur in a different stream than the previous cue (i.e., the probability that the cue was a shift cue was 0.86 ); while in a third set, stay and shift cues were equally likely.

This design controlled or eliminated several processes that often accompany stimulus-driven shifts of spatial attention. First, effects of stimulus-driven reorienting were measured independently of expectation, since activations were measured when shifts of attention were expected or unexpected. In a previous report involving this data set (Shulman et al., 2009), we showed that expectation and stimulus-driven reorienting had independent effects on the BOLD signal in TPJ but strongly interacting effects in regions of frontal cortex, anterior insula, and basal ganglia. Second, because activations during reorienting were measured to a cue that indicated where but not when a target would appear, time-locked activations related to temporal prediction of target onset were eliminated (Coull and Nobre, 1998; Coull et al., 2000), including those that involved a heightened state of alertness or motor preparation. Third, because activations during reorienting were measured to cues rather than to targets, the effects of processes related to target detection, such as response selection and execution and performance monitoring, were eliminated. Conversely, the experimental design allowed an assessment of hemispheric asymmetries evoked by the detection of targets in both visual fields at the currently attended location, eliminating effects of spatial reorienting.

Stimulus-driven shifts of spatial attention can potentially involve both goal-driven and automatic (i.e., exogenous) components, the latter isolated by measuring shifts of attention evoked by cues that have high sensory salience but are noninformative about the target (Posner and Cohen, 1984). However, the cues in the present experiment were $100 \%$ valid. Previous studies have shown that TPJ activations during stimulusdriven shifts of attention reflect goal-driven much more than exogenous processes (Downar et al., 2001; de Fockert et al., 2004; Kincade et al., 2005; Serences et al., 2005; Indovina and Macaluso, 2007) (for review, see
Corbetta et al., 2008). Therefore, the strong TPJ activations observed in the present paradigm (Shulman et al., 2009) likely also reflected goaldriven processes.

The present study involved a reanalysis of an experiment that involved the above paradigm and has previously been published (Shulman et al., 2009), and focused specifically on the presence of hemispheric asymmetries for stimulus-driven shifts of spatial attention and target detection.

\section{Subjects and stimuli}

Twenty-four right-handed subjects gave informed consent in accordance with guidelines set by the Human Studies Committee of Washington University. Data were eliminated from two subjects due to eye movements and from one subject due to movement artifacts.

Stimuli were presented with a Power Macintosh G4 computer (Apple) using Matlab software (MathWorks) with the psychophysics toolbox (Brainard, 1997; Pelli, 1997). Images were projected to the head of the bore of the scanner via an LCD projector (Sharp LCD C20X) and viewed with a mirror attached to the head coil.

Four RSVP streams of colored drawings of inanimate objects were presented in the left visual field and four in the right visual field (Fig. 1). The target streams, which could potentially contain the target object, were surrounded by three nontarget streams that contained only distracter objects. One nontarget stream was positioned above the target stream, one was below, and a third was more eccentric. The target streams were located at an eccentricity of $5^{\circ}$. All objects in both target and nontarget streams were $\sim 3^{\circ}$ by $3^{\circ}$. Distracter objects were sampled from a population of 40 objects and target objects were sampled from a separate population of 12 objects. The cue was a filled red square, $3^{\circ}$ by $3^{\circ}$, located in the target stream. None of the objects were red and when the cue was presented, no other objects were present in the field.

The discriminability of the target object was adjusted by adding colored noise to each object in the target streams in both fields (i.e., noise was added to both potential target streams, not only to the stream that was presently cued). To create the noise, a percentage of the pixels defining the target stream location in each field were randomly colored, with each pixel displayed in one of five randomly selected hues.

\section{Procedure}

Subjects were shown the target object, randomly selected from the 12 objects designated as targets, before each scan. The target object for a scan never appeared as a nontarget object in another scan. At the start of a scan, subjects fixated a central cross for $41.2 \mathrm{~s}$. A cue then appeared at one of the two target stream locations, indicating the initial stream to be attended. The eight RSVP streams then appeared. Each display frame of 8 objects was presented for $120 \mathrm{~ms}$, with no interstimulus interval (ISI) separating it from the next frame. Each cue appeared for $160 \mathrm{~ms}$, again with a $0 \mathrm{~ms}$ ISI before the next display frame. Subjects pressed a magnetic resonance (MR) compatible button when they detected a target. Target objects only appeared in the cued stream. A target occurred with a fixed probability independently in each $1.08 \mathrm{~s}$ interval such that on average a target occurred about once every $10.5 \mathrm{~s}$. To allow separate button presses to be recorded for each target, the minimum intertarget interval was restricted to $1 \mathrm{~s}$. All responses were made with the right hand. Cues occurred on average every $2.06,4.12$, or $6.18 \mathrm{~s}$ within a temporal window of plus or minus $400 \mathrm{~ms}$ centered on those values. Cue onset and target onset were independent except that a target could not occur simultaneously with a cue or in the $120 \mathrm{~ms}$ display frame preceding a cue. Therefore, cues provided spatial information about targets but essentially no temporal information. Following the initial $41.2 \mathrm{~s}$ fixation period, the stimulus display was presented for $185.4 \mathrm{~s}$ and was followed by a $30.9 \mathrm{~s}$ fixation period, resulting in a scan duration of $257.5 \mathrm{~s}$.

Each subject received 16 scans. In six scans the probability of a stay cue was 0.14 (shift cue probability was 0.86 ), in four scans, stay cue probability was 0.5 (shift cue probability, 0.5 ), and in six scans stay cue probability was 0.86 (shift cue probability, 0.14). Subjects were informed before each scan that it was "mostly stay," "mostly shift," or "stay and shift equally likely."

Before the scanning session, each subject received a practice session in which the noise level (percentage of voxels randomly colored) was deter- 
mined at which the task could be performed with a $60 \%$ target hit rate. This fraction was decreased by 0.05 for the session in the scanner, since our experience was that subject performance was slightly worse in the scanner. During the scanning session, the fraction was occasionally varied for each subject to approximately maintain performance at a desired level, using the following rules: if \%hits $<10$ on a scan, the noise fraction was lowered by 0.1 ; if $\%$ hits $<40$ on two consecutive scans, the noise was lowered by 0.1 ; if \%hits $<50$ on three consecutive scans, the noise was lowered by 0.05 ; if $\%$ hits $>90$ on a scan, the noise fraction was raised by 0.05 ; if $\%$ hits $>80$ on two consecutive scans, the noise was raised by 0.05 ; if $\%$ hits $>70$ on three consecutive scans, the noise was raised by 0.05 .

\section{Eye movement recording}

To verify that subjects followed instructions to remain fixated, each subject's eyes were carefully monitored via a camera on all scans. Moreover, eye movements were recorded for each subject using an infrared eye-tracking system on some scans during the scanner session (22 subjects, ISCAN ETL-200) or during a behavioral session outside of the scanner (2 subjects, ASL 504). Eye movement data were lost for one subject because of technical difficulties.

\section{Functional magnetic resonance}

imaging methods

Image acquisition. MR imaging (MRI) scans were collected on a Siemens Allegra 3T scanner, using a gradient echo EPI sequence to measure blood oxygenation-level-dependent (BOLD) contrast. Thirty-two contiguous 4 $\mathrm{mm}$ slices were acquired, $4 \times 4 \mathrm{~mm}$ in-plane resolution, echo time $(\mathrm{TE})=25 \mathrm{~ms}$, flip angle $=90^{\circ}$, repetition time $(\mathrm{TR})=2.06 \mathrm{~s}$. A sagittal MP-RAGE T1-weighted image $(\mathrm{TR}=$ $1810 \mathrm{~ms}, \mathrm{TE}=3.93 \mathrm{~ms}$, flip angle $=12^{\circ}$, inversion time $=1200 \mathrm{~ms}$, voxel size $=1 \times 1 \times 1.25$ $\mathrm{mm}$ ) and a transverse turbo spin-echo T2weighted image $(\mathrm{TR}=8430 \mathrm{~ms}$, $\mathrm{TE}=96 \mathrm{~ms}$, flip angle $=180^{\circ}$, voxel size $=1.1 \times 1.1 \times 3.0$ $\mathrm{mm}$ ) were also collected.

Preprocessing. Asynchronous slice acquisition was compensated by sinc interpolation so that all slices were aligned to the start of the frame. A whole-brain normalization corrected for changes in signal intensity across scans. Data were realigned within and across scans to correct for head movement. Atlas space images were created using the following procedure. For each subject, an EPI image based on the average of the first frame of each functional run was registered to the subject's T2-weighted structural image, which in turn was registered to the subject's T1-weighted MP-RAGE. Both registrations were accomplished using a cross-modal procedure based on aligning image gradients (Rowland et al., 2005). The MP-RAGE was then transformed to an atlas-space (Talairach and Tournoux, 1988) representative target using a 12-parameter affine transformation. The atlas space representative target brain consisted of coregistered MP-RAGE images acquired in 12 normal adults and was put into Talairach space using the method of Lancaster et al. (1995). The EPI- $>$ T2W->MPRAGE- $>$ atlas et al. (2009), their Figure 1.

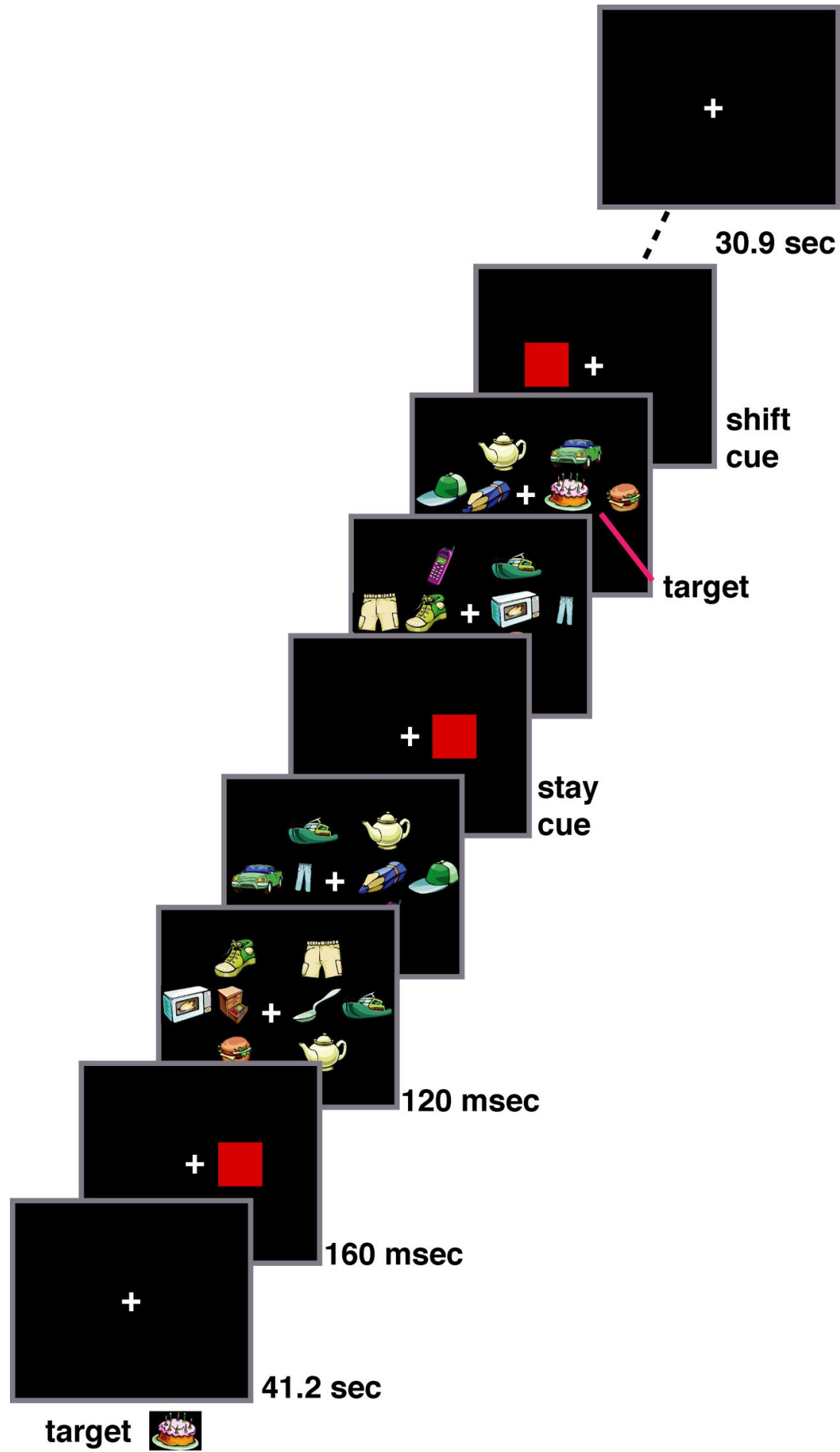

Figure 1. Procedure. The target object to be detected was presented at the beginning of the scan. Following a fixation period $(41.2 \mathrm{~s}$ ), a red square ( $160 \mathrm{~ms}$ duration) cue indicated the RSVP stream in which targets would appear. Subsequent cues indicated targets would continue to appear in the currently attended stream (stay cues) or in the opposite stream (shift cues). Successive cues were separated by $2.06,4.12$, or $6.18 \mathrm{~s}$ while successive targets were separated on average by $\sim 10.5 \mathrm{~s}$. The scan ended following a post-task fixation period (30.9s). See Materials and Methods for details. This figure appeared previously in the study by Shulman

transformations were composed and applied to the subject's data-space EPI images to create atlas-space EPI images resampled to $3 \mathrm{~mm}$ isotropic voxels. Movement correction and atlas transformation was accomplished in one resampling step to minimize blur and noise.

Statistical analysis of significant activations in each hemisphere. The BOLD signal during the $185.6 \mathrm{~s}$ task period was analyzed with the general 
linear model (GLM). Four cue regressors (stay left, stay right, shift left, and shift right), each consisting of 10 separate time point regressors that estimated the hemodynamic response out to $18.5 \mathrm{~s}$ without assuming a response shape (Ollinger et al., 2001), were estimated for each probability condition (i.e., stay cue probability $=0.14,0.50$, and 0.86 ). Four sets of target time point regressors, not separated by cue probability, were also estimated: detected target left and right, missed target left and right. In addition, regressors were included for baseline, linear trend and lowfrequency components $(<.009 \mathrm{~Hz})$ of the BOLD signal in each scan.

The resulting whole-brain maps of time courses for cues and targets were analyzed by conventional thresholded methods (Shulman et al., 2009) that identified the significant activations in each hemisphere related to the experimental variables. In particular, the time course maps were spatially smoothed by a Gaussian filter with a full-width-at-halfmaximum of $6 \mathrm{~mm}$ and analyzed by ANOVAs in which subject was treated as a random effect.

Statistical comparison of activations in left and right hemispheres. Several papers have discussed problems with conventional methods for assessing hemispheric differences in BOLD activity (Liégeois et al., 2002; Stevens et al., 2005; Macaluso and Patria, 2007). Hemisphere differences cannot be inferred from finding a significant activation in one hemisphere but not in the other, since this result may be an artifact of thresholding. Conversely, the presence of significant activations of both hemispheres is fully compatible with hemispheric differences, since the activations may differ in magnitude. Approaches based on comparing the number of significantly activated voxels in specific regions from each hemisphere are highly dependent on thresholds and decisions concerning which regions are homologous.

These considerations indicate the importance of conducting direct and unbiased statistical comparisons of the two hemispheres to identify activations that are significantly lateralized. In several recent studies, voxelwise comparisons across the hemispheres have been conducted after normalizing images to a symmetric EPI template (Stevens et al., 2005; Macaluso and Patria, 2007). However, since left and right hemispheres are not mirror images, we adopted a slightly different approach (see supplemental Fig. 1, available at www.jneurosci.org as supplemental material). For each subject, two sets of atlas-space GLMs were created. The first set of GLMs was identical to the set described in the previous section. To create the second set of GLMs, each subject's data-space EPI image was flipped across the midline and registered to the subject's own unflipped EPI image using a 12-parameter affine transformation. As a result, the subject's left (right) hemisphere was transformed in the best fitting manner to the subject's right (left) hemisphere. Following this step, the previously computed transformations from the unflipped EPI image to the structural images (T2-weighted, MP-RAGE) and from the MP-RAGE to the atlas representative target could be applied. "Flipped" GLMs were then computed from these flipped atlas-space EPI images. Finally, a voxelwise ANOVA was conducted in which time courses from the flipped and normal (unflipped) GLMs were compared. In this ANOVA (see supplemental Fig. 1, available at www.jneurosci.org as supplemental material), there were two separate voxelwise comparisons in atlas space: (1) each voxel in the unflipped right hemisphere (RH) was compared to the voxel in the left hemisphere (LH) that had been flipped and transformed to that same right hemisphere voxel, and (2) each voxel in the unflipped left hemisphere was compared to the voxel in the right hemisphere that had been flipped and transformed to that same left hemisphere voxel. Typically, if activations were larger in an unflipped RH voxel than in the corresponding flipped $\mathrm{LH}$ voxel, then a flipped $\mathrm{RH}$ voxel also showed larger activations than the corresponding unflipped LH voxel. Therefore, the flipping procedure itself did not introduce a bias that favored either the flipped or unflipped hemisphere. Any exceptions to this rule are noted in Results. For clarity, unless noted, all figures in Results show regions in which the activation at the unflipped voxel was greater than the activation from the corresponding flipped voxel.

All voxelwise ANOVAs were corrected for nonindependence of time points by adjusting the degrees of freedom and corrected over the brain for multiple comparisons using joint $z$-score/cluster size thresholds (Forman et al., 1995) corresponding to $z=3.0$ and a cluster size of 13 voxels with contiguous edges. The $z$-score/cluster size thresholds were determined using volume-based monte-carlo simulations.

For purposes of plotting time courses and reporting atlas-space coordinates, regions of interest were automatically created from the voxellevel ANOVA maps using a peak-finding routine (Kerr et al., 2004). For display purposes, volumes were mapped to surface-based representations using the PALS atlas and CARET software (Van Essen, 2005).

\section{Results}

\section{Behavior}

Subjects detected on average $61.7 \%$ of targets, with a mean false alarm rate (number of false alarms/number of targets) of $4.7 \%$. The detection rate was well below ceiling, indicating that the task was quite difficult, but also well above the false alarm rate, indicating above chance performance. Mean correct reaction time (RT) was $596 \mathrm{~ms}$. A full analysis of the behavioral data has been separately reported (Shulman et al., 2009).

An analysis of eye movements, which has been separately reported (Shulman et al., 2009), indicated that two subjects showed poor fixation. These subjects were excluded from analyses of the behavioral and imaging data.

\section{Functional MRI}

We first determined whether stimulus-driven shifts of spatial attention produced activations that were right hemisphere dominant. Our prior work on this dataset (Shulman et al., 2009) had shown that stimulus-driven shifts of spatial attention activated bilateral dorsal frontoparietal cortex and right (R) TPJ, as defined by greater responses to shift cues than stay cues. Some of the dorsal frontoparietal regions [e.g., bilateral precuneus and frontal eye field (FEF)], as well as R TPJ, showed transient responses to cues (shift signals) that did not strongly depend upon the location of the cue. These regions may be involved in shifting spatial attention (Kelley et al., 2008). A second set of dorsal frontoparietal regions [medial intraparietal sulcus (IPS) and precentral/FEF) showed sustained responses to the cue that were much stronger for contralateral than ipsilateral cues. These regions may be involved in maintaining spatial attention at a specific location. The medial IPS regions were similar to IPS regions that have been shown to have topographic maps of the contralateral visual field (Sereno et al., 2001; Silver et al., 2005; Swisher et al., 2007) and were anatomically distinct from the precuneus regions that showed the most robust shift signals.

\section{Transient shift signals for stimulus-driven shifts of attention:} right-lateralized in $R$ TPJ, bilateral in dorsal frontoparietal cortex We first examined whether regions showing transient shift signals were asymmetrically activated. Figure $2 \mathrm{~A}$ shows the results of a voxelwise analysis that tested for a significant hemispheric asymmetry in voxels that differentially responded to stay and shift cues, as assessed by the interaction of Hemisphere (flipped, unflipped) by Cue Type (shift, stay) by Time (10 time points). Each voxel in the left (right) hemisphere was compared to a voxel in the right (left) hemisphere that had been flipped over the midline and then transformed to the left (right) hemisphere (see methods for details). A large activation was observed in TPJ (52, $-48,14 z=4.64 ; 46,-45,26 z=4.59$ ) (Table 1), indicating a significant hemispheric difference in stimulus-driven reorienting. The time courses in Figure $2 \mathrm{~A}$ confirmed the presence of larger activations in right TPJ than left TPJ. Figure $2 B$ shows conventional thresholded ANOVA maps of voxels in the left and right hemispheres that differentially responded to stay and shift cues [Cue Type (shift, stay) by Time, corrected for multiple com- 
parisons]. A clear difference between left and right hemispheres was evident in the region of TPJ.

In contrast to the strong right hemisphere dominance in TPJ, however, the direct comparison of left and right hemispheres (Fig. 2A) yielded no significant hemispheric asymmetries in dorsal frontoparietal cortex. The only other significant foci in the entire image [Hemisphere (flipped, unflipped) by Cue Type (shift, stay) by Time (Table 1)] were located in $\mathrm{R}$ posterior ventral parieto-occipital cortex $(14,-57,23 z=3.80)$ and in left (L) Caudate $(-24,12,15 z=4.11)$. Moreover, the latter region was only significant in the comparison involving unflipped LH voxels and flipped RH voxels, not in the analogous comparison of flipped LH voxels and unflipped RH voxels, indicating that the asymmetry was not robust. While the thresholded maps in Figure $2 B$ suggested that some parietal regions might show stronger activations in one hemisphere or the other, these trends did not survive the direct statistical comparison shown in Figure $2 \mathrm{~A}$ and Table 1. These results emphasize that rigorous assessments of hemispheric differences require direct statistical comparisons.

Importantly, right hemisphere dominance of R TPJ during stimulus-driven reorienting was observed both for the comparison in which unflipped right hemisphere voxels showed greater activations than the corresponding flipped left hemisphere voxels (as in Fig. 2A) and for the analogous comparison in which flipped right hemisphere voxels showed greater activations than the corresponding unflipped left hemisphere voxels (see supplemental Fig. 2, available at www.jneurosci.org as supplemental material). Therefore, the flipping procedure itself did not introduce a bias that favored either the flipped hemisphere or the unflipped hemisphere.

\section{Sustained spatially selective attentional modulations: bilateral in dorsal frontoparietal cortex}

Figure $3 A$ displays the regions in medial IPS that showed sustained and spatially selective attentional modulations, as indicated by a significant interaction of Cue Type (shift, stay) by Cue Location (left, right) by Time, corrected for multiple comparisons. Because the ANOVA compared stay and shift cues that were presented at the same location, controlling for purely sensory activations, the activations reflected attentional modulations (Shulman et al., 2009). The time courses in the graphs confirmed that the activations were highly spatially selective and were sustained over time (see (Shulman et al., 2009) for the spatially selective time courses in FEF). While the time courses appeared to show a deactivation for ipsilateral cues, this deactivation was relative to a baseline that included both sensory-evoked and taskevoked activity. Spatially selective parietal regions that show a stronger response for contralateral than ipsilateral stimuli nevertheless show a substantial response to the ipsilateral stimuli (Jack et al., 2007).
Table 1. Cue-related regions showing significant hemispheric asymmetries in voxelwise ANOVAs

\begin{tabular}{lllll}
\hline Region & $x$ & $y$ & $z$ & $z$-score \\
\hline Cues: Hemisphere $\times$ CueType (shift, stay) $\times$ Time & & & & \\
$\quad$ R TPJ & 52 & -48 & 14 & 4.64 \\
& 46 & -45 & 26 & 4.59 \\
$\quad$ R ventral parieto-occipital & 14 & -57 & 23 & 3.80 \\
Cues: Hemisphere $\times$ CueType (shift, stay) $\times$ CueLocation & & & & \\
$\quad$ (contralateral, ipsilateral) $\times$ Time & & & & \\
$\quad$ R IPL & 42 & -58 & 42 & 3.65 \\
\hline
\end{tabular}

To determine whether the spatially selective contralateral advantage was the same in the two hemispheres, we recoded the left cue/right cue variable as contralateral cue/ipsilateral cue and directly compared the time courses for the unflipped and flipped voxels in an ANOVA [Hemisphere (flipped, unflipped) by Cue Location (contralateral, ipsilateral) by Cue Type (stay, shift) by Time]. This voxelwise ANOVA yielded almost no significant effects in dorsal frontoparietal cortex (Fig. $3 B$ ). No effects were observed in SPL, IPS, or FEF, consistent with the conventional thresholded images of the Cue Type by Cue Location by Time interaction in the left and right hemispheres (Fig. 3A), which qualitatively showed no evidence of right hemisphere superiority. A significant activation was only observed in a small region in $\mathrm{R}$ dorsal IPL (42, $-58,42 z=3.65$; Table 1$)$ and in L anterior precuneus $(-17,-41,46 z=3.57)$, and the latter region did not have a focus for the analogous flipped right hemisphere/unflipped left hemisphere image comparison. 


\section{A Shift vs Stay by Cue Location}

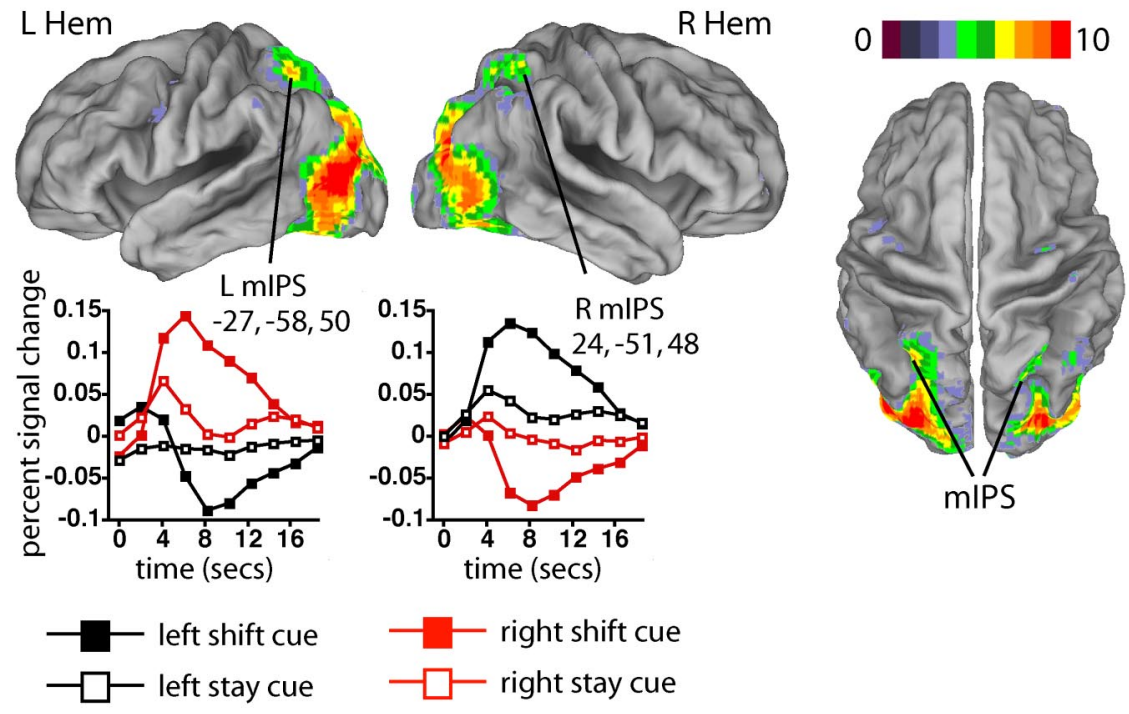

\section{B Shift vs Stay by Cue Location: R Hem > L Hem}
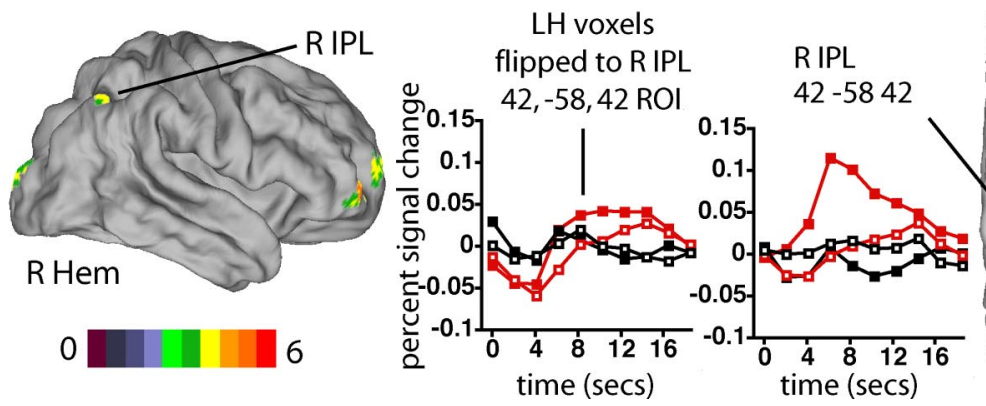

Figure 3. A, Lateral and dorsal views of voxels from separate thresholded statistical maps of the left and right hemispheres that showed a spatially selective attentional modulation, i.e., activations in which the difference between shift and stay cues significantly depended on cue location [from the ANOVA map of significant voxels for the interaction of Cue Location (left, right) by Cue Type (shift, stay) by Time, corrected for multiple comparisons]. The color bar indicates the equivalent $z$-score for the $p$ value from the ANOVA. The graphs show the time courses of the activations in L IPS and R IPS as a function of Cue Type and Cue Location. Parts of $\boldsymbol{A}$ previously appeared in parts of Figure 4 in the study by Shulman et al. (2009). $\boldsymbol{B}$, Lateral and dorsal views of voxels in which the spatially selective attentional modulation showed a hemispheric asymmetry (from the ANOVA map of significant voxels for the interaction of Hemisphere [flipped, unflipped) Cue Location (left, right) by Cue Type (shift, stay) by Time, corrected for multiple comparisons]. The assignment of cue locations to left and right in the ANOVA was reversed in the flipped images to allow coding of contralateral and ipsilateral). Within dorsal frontoparietal cortex, only a small region in right IPL was significant. The color bar indicates the equivalent $z$-score for the $p$ value from the ANOVA. The graphs show time courses for this right IPL region and the corresponding flipped voxels from the left hemisphere. The region showed a preference for ipsilateral shift cues and was separate from the medial IPS region that showed the strongest attentional modulations in the thresholded images of Figure $3 A$. mIPS, Medial IPS; LH, left hemisphere; $\mathrm{RH}$, right hemisphere; Hem, hemisphere.

An interesting aspect of the dorsal IPL focus was that it showed larger activations for ipsilateral than contralateral shift cues (Fig. 3B), consistent with the hypothesis that the right hemisphere contains separate representations of the left and right visual fields. However, this intriguing result should be treated cautiously until it is replicated. Many studies of visual field preferences and polar-angle topography in parietal cortex have been conducted, but to our knowledge none have reported parietal regions showing ipsilateral preferences [(Sereno et al., 2001; Schluppeck et al., 2005; Silver et al., 2005; Hagler et al., 2007; Jack et al., 2007; Molenberghs et al., 2007; Serences and Yantis, 2007; Swisher et al., 2007; Sylvester et al., 2007; Saygin and Sereno, 2008) although Siman-Tov et al. (2007) reported an overall left visual field advantage in many brain regions, including IPS], and
R Hem

the IPL focus was clearly lateral to the medial IPS regions that showed the strongest spatially selective attentional modulations (Fig. $3 A$ ). Moreover, the time course of the IPL activation showed only a weak decrease for contralateral shift cues relative to stay cues and no clear differences between ipsilateral and contralateral stay cues, unlike the foci in medial IPS, raising questions about its robustness.

To summarize, dorsal frontoparietal regions that were transiently activated by stimulus-driven reorienting or that showed sustained spatially selective attentional modulations were largely bilateral. However, TPJ, which showed a robust advantage for shift cues relative to stay cues in both visual fields, showed strong right hemisphere dominance. These results indicate that during stimulus-driven reorienting, right hemisphere dominance does not occur within the dorsal network that primarily controls reorienting, but only within a second, ventral network that is coactivated with the dorsal network.

Importantly, the hemispheric asymmetry in TPJ activity was demonstrated using an experimental design that controlled or eliminated several processes that often accompany stimulus-driven shifts of spatial attention. The activations from shifting attention to an unattended stimuli were separated from the activations caused by a breach of expectation, were measured to cues rather than to targets (e.g., invalid targets), eliminating the effects of response-related and other target-related processes such as performance monitoring, and involved cues that provided spatial but not temporal information about targets.

\section{Target detection: widespread right hemisphere dominance}

Both dorsal and ventral frontoparietal cortex, along with other regions, was activated during target detection and response (supplemental Fig. 3, available at www.jneurosci.org as supplemental material). While dorsal frontoparietal regions did not show hemispheric asymmetries during stimulusdriven shifts of spatial attention, they may nevertheless show asymmetries during detection, reflecting an involvement in target-related processes that are asymmetrically organized. We tested whether hemispheric asymmetries were observed for detected targets, as indexed by the interaction of Hemisphere (flipped, unflipped) by Time in the event-related time courses for detected targets. Because targets only occurred in the attended stream, hemispheric asymmetries during target detection were assessed under conditions in which the target did not evoke a shift of spatial attention.

Since subjects responded with their right hand, left hemisphere dominant activations were expected in standard somato- 


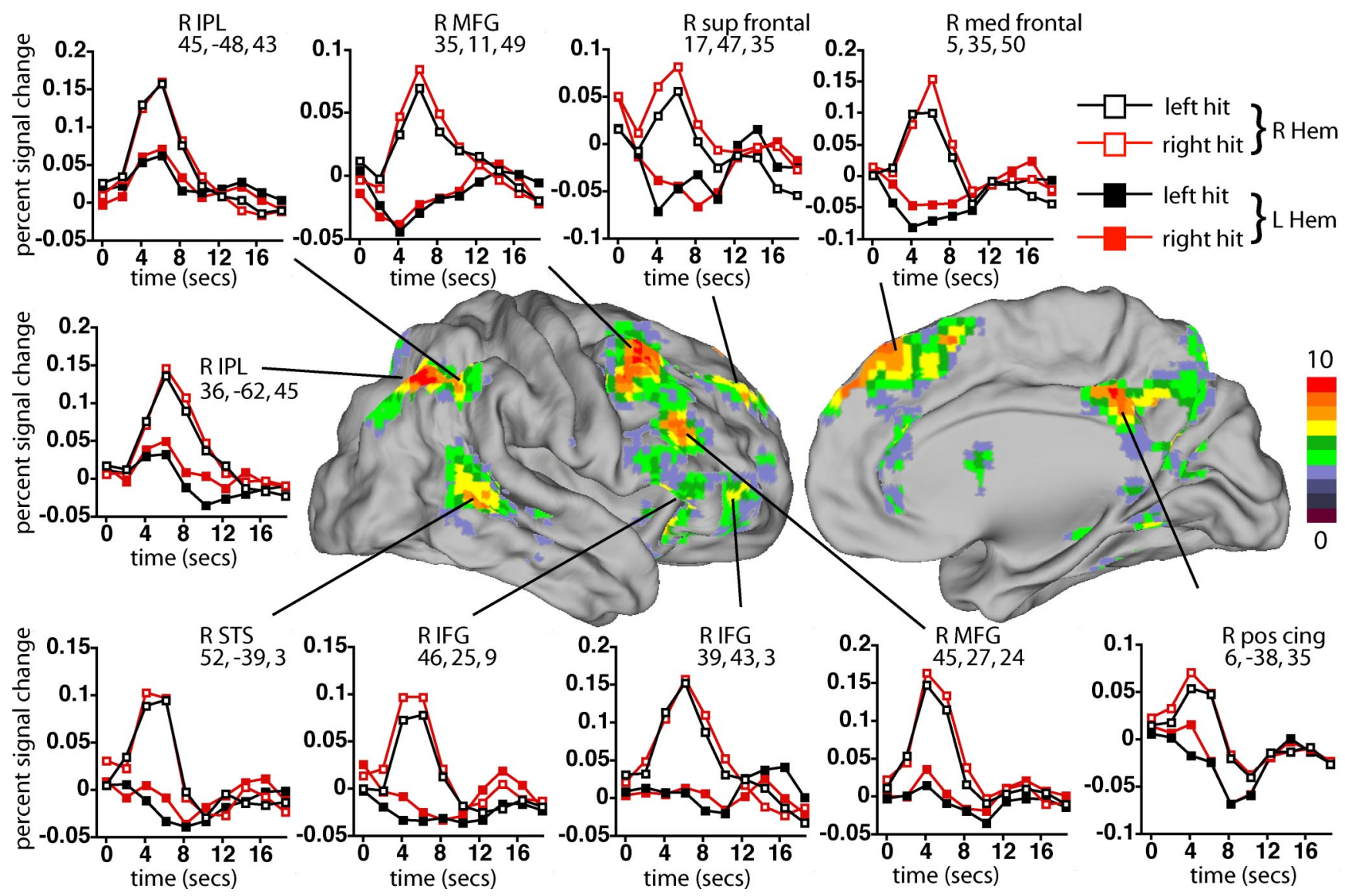

Figure 4. Right hemispheric dominant activations evoked by detection of targets, from the group Hemisphere by Time ANOVA map, corrected for multiple comparisons. The color bar indicates the equivalent $z$-score for the $p$ value from the ANOVA. The statistical maps were masked so that right hemisphere (unflipped) voxels were only included if their activation magnitudes were positive. The magnitude corresponding to the time course at a voxel was computed using a standard hemodynamic response function (Boynton et al., 1996). The color bar indicates the equivalent $z$-score for the $p$ value from the ANOVA. The graphs show the time course of activation to cues for right hemisphere voxels (unflipped) and the corresponding left hemisphere voxels (flipped), broken down by target visual field. sup, Superior; med, medial; pos, posterior; cing, cingulate; Hem, hemisphere.

motor regions (e.g., left central sulcus, postcentral gyrus, or left SMA). Supplemental Figure 4 (available at www.jneurosci.org as supplemental material) shows that left hemisphere dominant activations were almost entirely confined to these regions. One possible exception in left occipitotemporal cortex may have corresponded to the extrastriate body area (Downing et al., 2001), which is activated by motor responses (Astafiev et al., 2004).

In contrast, Figure 4 shows that right hemisphere dominance during target detection was observed in many parietal, temporal, and prefrontal regions, including inferior parietal lobule (IPL), superior temporal sulcus (STS), inferior frontal gyrus (IFG), middle frontal gyrus (MFG), and superior frontal regions (Table 2). The graphs in Figure 4, which show the time courses broken down by target location, indicate that right hemisphere dominance was present regardless of the visual field of the target.

Figure 5 shows that the temporal lobe activations included the TPJ region that was right hemisphere dominant for stimulusdriven shifts of spatial attention, although the overall distribution of the asymmetric cue and target activations in the temporal lobe appeared to differ. Therefore, TPJ showed right hemisphere dominance both during stimulus-driven shifts of spatial attention and during target detection at an attended location.

The extensive right hemisphere dominance for target detection did not reflect a methodological bias for the right hemisphere since as noted above, strong left hemisphere dominance was observed in the expected somatomotor regions given the right hand detection response (supplemental Fig. 4, available at www.jneurosci.org as supplemental material). The almost complete right hemispheric dominance outside of classical somatomotor regions also cannot be explained by misaligned or inappropriate correspondences between voxels in the left and right hemispheres, since misalignment would have produced paired left and right hemisphere dominant activations in adjacent regions. Moreover, these results are consistent with those of Stevens et al. (2005), who conducted direct voxelwise comparisons of left and right hemispheres and reported right hemisphere dominance during the detection of auditory oddball targets in several frontal, parietal, and temporal regions outside of classical somatomotor regions.

\section{Target detection-related asymmetries: poor overlap with dorsal} frontoparietal regions

The strong right hemisphere dominance for target detection was not centered on the dorsal frontoparietal network activated by shifts of spatial attention. As discussed earlier, the transient component of the dorsal attention network was defined by the difference between shift and stay cues while the sustained spatially selective component was defined by the interaction of the shift vs stay difference with cue location (Figs. 2, 3).

Figure 6A compares dorsal, lateral, and medial views of the parietal regions comprising the transient (left panel) and sustained (middle panel) components of the dorsal attention network with the 
Table 2. Target-related regions showing significant right hemispheric dominance in voxelwise ANOVAs

\begin{tabular}{|c|c|c|c|c|}
\hline \multirow[b]{2}{*}{ Region } & \multicolumn{4}{|c|}{ Targets: Hemisphere (left, right) $\times$ Time } \\
\hline & $x$ & $y$ & $z$ & $z$-score \\
\hline R cuneus & 14 & -60 & 14 & 6.79 \\
\hline \multirow[t]{2}{*}{ R dorsal IPL } & 36 & -62 & 45 & 9.40 \\
\hline & 45 & -48 & 43 & 8.02 \\
\hline Rprecuneus & 10 & -65 & 55 & 4.08 \\
\hline \multirow[t]{2}{*}{ R posterior cingulate } & 6 & -38 & 34 & 8.59 \\
\hline & 10 & -62 & 37 & 5.94 \\
\hline R retrosplenial & 6 & -46 & 0 & 5.69 \\
\hline RSTS & 52 & -39 & 3 & 8.72 \\
\hline \multirow[t]{2}{*}{ R superior frontal } & 22 & 10 & 56 & 6.28 \\
\hline & 17 & 47 & 35 & 7.68 \\
\hline \multirow[t]{5}{*}{ R MFG } & 35 & 11 & 49 & 9.40 \\
\hline & 45 & 27 & 24 & 8.14 \\
\hline & 44 & 12 & 33 & 5.88 \\
\hline & 37 & 28 & 42 & 5.27 \\
\hline & 25 & 33 & 45 & 4.85 \\
\hline \multirow[t]{4}{*}{ R medial prefrontal } & 5 & 35 & 50 & 8.09 \\
\hline & 4 & 20 & 57 & 7.34 \\
\hline & 3 & 46 & 41 & 6.73 \\
\hline & 19 & 55 & 16 & 5.07 \\
\hline \multirow[t]{4}{*}{ Anterior cingulate } & 4 & 23 & 45 & 6.48 \\
\hline & 10 & 32 & 20 & 5.99 \\
\hline & 6 & 37 & 1 & 4.93 \\
\hline & 4 & 35 & 29 & 4.77 \\
\hline \multirow[t]{4}{*}{ RIFG } & 45 & 25 & 9 & 8.05 \\
\hline & 39 & 19 & -7 & 7.70 \\
\hline & 39 & 43 & 3 & 6.17 \\
\hline & 29 & 32 & -7 & 5.46 \\
\hline R caudate & 11 & 10 & 6 & 6.65 \\
\hline
\end{tabular}

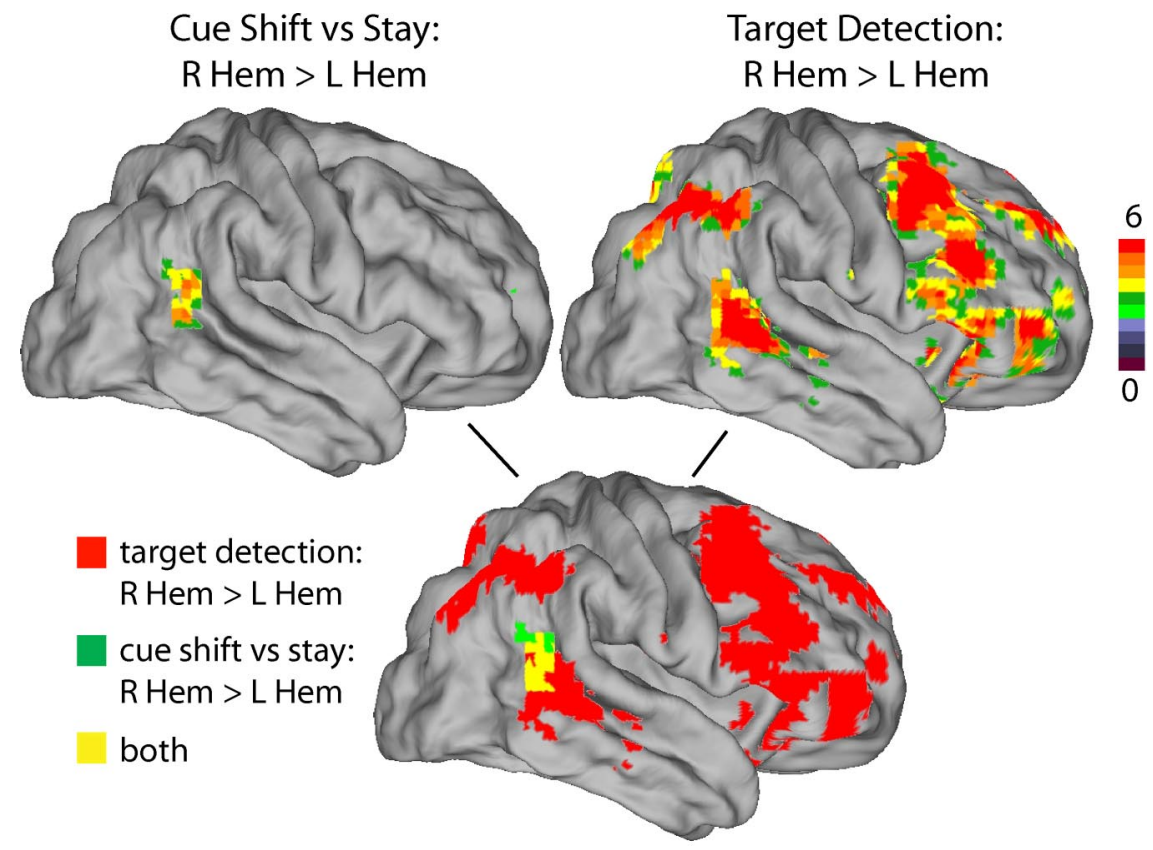

Figure 5. Comparison of right hemispheric dominant activations for stimulus-driven reorienting and target detection. The images in the top row show ANOVA maps of voxels that showed significant right hemisphere dominance during stimulus-driven reorienting, as assessed by the multiple-comparison corrected map for Hemisphere by Cue Type (shift, stay) by Time (for cues), and voxels that showed significant right hemisphere dominance during target detection, as assessed by the multiple-comparison corrected map for Hemisphere by Time (for detected targets). The color bar indicates the equivalent $z$-score for the $p$ value from the ANOVA. The bottom figure indicates those voxels that were significantly present in one (red, green voxels) or both (yellow voxels) maps. Hem, Hemisphere. parietal regions showing significant right hemisphere dominance during target detection (right panel). A clear dissociation was evident between transient and sustained components of the dorsal parietal attention network and right hemisphere dominant detection-related regions. Transient activations due to stimulusdriven reorienting were observed most strongly in dorsal anterior precuneus (medial view) and SPL (dorsal view), and sustained activations were observed most strongly in medial IPS (dorsal and lateral views), while hemispheric asymmetries during target detection were observed most strongly in regions of IPL (dorsal and lateral view). Within dorsal frontal cortex (Fig. $6 B$ ), activations due to stimulus-driven shifts of spatial attention were observed most strongly in FEF/precentral regions, while hemispheric asymmetries during detection were observed most strongly in dorsolateral prefrontal cortex.

Therefore, while hemispheric asymmetries during target detection were observed in some parietal regions that were modestly or weakly activated by shifts of spatial attention (e.g., IPL, including the focus that showed stronger responses to ipsilateral than contralateral shift cues, and posterior SPL/precuneus), the strongest detection-related asymmetries occurred in regions outside the dorsal attention network, and conversely, the dorsal frontoparietal voxels most strongly activated during stimulusdriven shifts of spatial attention (e.g., anterior precuneus, medial IPS, and FEF) did not show target detection-related hemispheric asymmetries.

The weak lateralization of activations in the dorsal frontoparietal network during target detection did not reflect an absence of activation. Supplemental Figure 3 (available at www.jneurosci.org as supplemental material), for example, indicates that robust detection-related activity was observed in FEF and precuneus. Rather, regions within the network were significantly activated during detection but did not generally show a hemispheric asymmetry.

The overall conclusion is that while strong right hemisphere dominance was observed during stimulus-driven shifts of spatial attention and during target detection, these hemispheric asymmetries largely occurred in regions outside the dorsal frontoparietal network.

\section{Discussion}

The dorsal frontoparietal network plays a primary role in selective attention to the environment (Kastner and Ungerleider, 2000; Corbetta and Shulman, 2002). The present experiment directly tested for hemispheric asymmetries in this network during stimulus-driven reorienting and found little evidence of right hemisphere dominance, either in transient or in sustained spatially selective components of the network. Furthermore, hemispheric asymmetries were largely absent during the detection of an infrequent, poorly discriminable target at the attended location, indicating that the dorsal network was symmetrically activated across multiple task processes.

An important caveat, however, is that while left and right dorsal frontoparietal 
regions were equivalently activated during shifts of spatial attention, the functional roles of those activations may differ (Mevorach et al., 2009; Ruff et al., 2009). For example, a recent study involving concurrent TMS-functional MRI (fMRI) reported that TMS of right but not left parietal cortex evoked BOLD signals in V1 through V4 and MT+ (Ruff et al., 2009). Similarly, TMS and conjoint TMS-fMRI studies have explored whether one hemisphere can compensate for impaired functioning of the other hemisphere (Sack et al., 2005; O'Shea et al., 2007).

TPJ is coactivated with the dorsal network during stimulus-driven shifts of spatial attention (Corbetta et al., 2008). While results from previous thresholded neuroimaging studies had qualitatively suggested that TPJ activation is right lateralized (Arrington et al., 2000; Corbetta et al., 2000; Downar et al., 2000; Macaluso et al., 2002), the present work provides the first quantitative evidence for this hypothesis [a prior study using direct voxelwise comparisons reported null results (Macaluso and Patria, 2007)].

TPJ also showed right hemisphere dominance during target detection, indicating it was asymmetrically activated across multiple task processes. TPJ activation is not restricted to stimulus-driven shifts of spatial attention, but occurs for a broad range of stimulus-driven transitions (Corbetta et al., 2008), including the transition from monitoring an RSVP stream to detection in the current study. The right hemisphere asymmetry in TPJ during both shifts of spatial attention and target detection supports a common switching function for this region.

\section{Right hemisphere dominance for target detection}

Target detection at the currently attended location in both visual fields produced widespread right hemisphere dominance that included not just the TPJ region asymmetrically activated by stimulusdriven reorienting, but also regions in STS, IPL, MFG, IFG, and dorsal anterior cingulate/preSMA that were largely outside the dorsal frontoparietal network. Therefore, right hemisphere dominance was observed during task processes that did not involve shifts of spatial attention, perhaps reflecting nonselective attentional processes such as alerting/arousal and capacity (Posner and Boies, 1971). Target detection increases capacity demands (Duncan, 1980) and likely produces transient changes in alerting/arousal, particularly when targets are infrequent. However, since capacity demands and alerting/arousal were not manipulated in the current study, their relationship to the hemispheric asymmetries observed during detection remains an open question.

Nevertheless, it is worth noting that thresholded neuroimaging studies have not yielded consistent evidence for lateralization of regions underlying target-related phenomena thought to reflect capacity limitations (for review, see Marois and Ivanoff, 2005), such as the attentional blink (but see Husain and Rorden, 2003) or psycho- logical refractory period. Both left and right hemisphere lesions produce decreases in quantitative measures of processing speed (Peers et al., 2005).

Conversely, many authors have suggested that alerting/ arousal is right hemisphere dominant [for reviews, see Posner and Petersen (1990) and Sturm and Willmes, 2001], both when an alert state is transiently established by a warning signal (Heilman et al., 1978; Heilman and Van Den Abell, 1980; Yokoyama et al., 1987) and when it is endogenously maintained (Dimond, 1979; Posner et al., 1987; Wilkins et al., 1987). Many of the asymmetric detection-related regions (e.g., MFG, IFG, IPL) observed here have previously been reported in thresholded neuroimaging studies concerned with the endogenous maintenance of an alert state (Pardo et al., 1991; Sturm et al., 1999). Therefore, while the current detection-related activations were strictly event-related and transient, similar regions might also be activated during sustained, endogenously maintained arousal. Alternatively, previous experiments may not have sufficiently controlled for activations from transient, detection-related processes evoked by hits (Sturm et al., 1999) or false alarms (Pardo et al., 1991; but see Foucher et al., 2004).

The widespread hemispheric asymmetries observed during target detection may partly reflect the involvement of diffusely 
projecting neuromodulatory systems such as the locus ceruleus/ noradrenaline (LC/NE) system, which has long been linked to alerting/arousal (Aston-Jones and Bloom, 1981a,b; Posner and Petersen, 1990). There is some evidence for a right hemisphere asymmetry of the LC/NE system (Posner and Petersen, 1990), primarily from experiments in which frontal lesions in rats decreased NE in cortex and LC (Robinson, 1979; Robinson and Coyle, 1980; Robinson, 1985) (for evidence in human thalamus, see Oke et al., 1978).

The LC/NE system has been proposed to facilitate transitions between behavioral states, including those related to shifts of attention and target detection (Bouret and Sara, 2005; Corbetta et al., 2008). Phasic LC/NE activity to detected targets during a taskengaged state has been hypothesized to "collapse" the different layers of task-relevant cortical networks so that a detected target can be quickly coupled to a motor response (Aston-Jones and Cohen, 2005). From these perspectives, the LC/NE system may have modulated the activity evoked in widely distributed right hemisphere regions during the transition from monitoring an attended RSVP stream to target detection/response and in the more limited right TPJ region during the stimulus-driven transition between selective attention to left and right RSVP streams.

\section{Implications for spatial neglect}

Theories of spatial neglect must explain several puzzling results. First, unilateral lesions that produce neglect often do not structurally damage the dorsal frontoparietal network (but see Molenberghs et al., 2008), yet this network primarily controls spatial orienting. Second, while the dorsal frontoparietal network is largely bilateral, spatial neglect is more profound following right hemisphere lesions (Heilman et al., 1985; Bowen et al., 1999; Ringman et al., 2004; Becker and Karnath, 2007). Third, hemispatial neglect often co-occurs with deficits that apply across the visual field ("nonlateralized" deficits), such as lowered arousal and capacity (Husain and Rorden, 2003).

The most prominent theory of neglect maintains that the left hemisphere controls shifts of attention to stimuli in the rightward direction, while the right hemisphere controls shifts of attention in either direction (Mesulam, 1981). Therefore, right hemisphere lesions leave only the left hemisphere system for orienting to the right visual field, while left hemisphere lesions produce less profound neglect since the remaining right hemisphere system can orient attention to both fields. The current work, however, in conjunction with earlier thresholded neuroimaging studies, indicates that spatially selective regions modulated by attention are largely bilateral and show a preference for contralateral stimuli. A small region in R IPL showed an ipsilateral preference, but the reliability of this result will need to be confirmed.

Lesions that produce neglect usually occur in regions ventral to the dorsal frontoparietal network, including insula, IFG, superior temporal cortex, and IPL (Vallar and Perani, 1986; Husain and Kennard, 1996; Karnath et al., 2001; Mort et al., 2003; Karnath et al., 2004; Corbetta et al., 2005). A second theory posits that these more ventral lesions structurally damage a right hemisphere dominant system that is not spatially selective and whose functions include stimulus-driven reorienting (Corbetta et al., 2005; He et al., 2007) and/or alerting/arousal (Robertson et al., 1997, 1998). In one account (Corbetta et al., 2005), this structural damage produces abnormal physiological responses in structurally undamaged dorsal parietal regions that directly control spatial attention (Corbetta et al., 2005; He et al., 2007). Reported abnormalities in dorsal parietal regions include greater taskevoked BOLD activity in left than right hemisphere regions
(Corbetta et al., 2005) and decreased interhemispheric restingstate functional connectivity (He et al., 2007). The mechanism by which structural damage to the right but not left hemispheres markedly disrupts the physiology of undamaged dorsal parietal regions is unclear but may involve disconnection phenomena (Bartolomeo et al., 2007). In one study, patients with the most severe spatial neglect showed structural damage in white matter tracts that may connect ventral and dorsal regions via MFG (He et al., 2007).

The present work shows that the right hemisphere regions most frequently lesioned in neglect patients, including IFG, superior temporal cortex, and IPL, also show significant right hemisphere dominance during target detection. It is unknown whether lesions to these regions impair physiological responses in undamaged right frontal regions that also show detection-related asymmetries (e.g., dorsal MFG and superior frontal cortex). Regardless, right hemisphere lesions that typically result in spatial neglect plausibly would impair target detection in both visual fields, consistent with some nonlateralized deficits that have been reported (Husain and Rorden, 2003). Therefore, in conjunction with the documented effects of these lesions in structurally undamaged dorsal parietal regions that control spatial attention (Corbetta et al., 2005; He et al., 2007), both lateralized and nonlateralized deficits would be expected. Interestingly, some studies indicate that right superior temporal lesions occur most frequently in neglect patients (Karnath et al., 2001, 2004). The present asymmetric activation of this region during both reorienting and target detection may well indicate a special role in such neglect-related phenomena as "disengagement" (Friedrich et al., 1998) and extinction. Therefore, the widespread right hemisphere dominance during target detection, as well as the more restricted lateralization of right TPJ during stimulus-driven reorienting, may partly explain the greater severity of neglect following right than left hemisphere strokes.

\section{References}

Arrington CM, Carr TH, Mayer AR, Rao SM (2000) Neural mechanisms of visual attention: object-based selection of a region in space. J Cogn Neurosci 12:106-117.

Astafiev SV, Stanley CM, Shulman GL, Corbetta M (2004) Extrastriate body area in human occipital cortex responds to the performance of motor actions. Nat Neurosci 7:542-548.

Aston-Jones G, Bloom FE (1981a) Nonrepinephrine-containing locus coeruleus neurons in behaving rats exhibit pronounced responses to nonnoxious environmental stimuli. J Neurosci 1:887-900.

Aston-Jones G, Bloom FE (1981b) Activity of norepinephrine-containing locus coeruleus neurons in behaving rats anticipates fluctuations in the sleep-waking cycle. J Neurosci 1:876-886.

Aston-Jones G, Cohen JD (2005) An integrative theory of locus coeruleusnorepinephrine function: adaptive gain and optimal performance. Annu Rev Neurosci 28:403-450.

Bartolomeo P, Thiebaut de Schotten M, Doricchi F (2007) Left unilateral neglect as a disconnection syndrome. Cereb Cortex 17:2479-2490.

Becker E, Karnath HO (2007) Incidence of visual extinction after left versus right hemisphere stroke. Stroke 38:3172-3174.

Bouret S, Sara SJ (2005) Network reset: a simplified overarching theory of locus coeruleus noradrenaline function. Trends Neurosci 28:574-582.

Bowen A, McKenna K, Tallis RC (1999) Reasons for variability in the reported rate of occurrence of unilateral spatial neglect after stroke. Stroke 30:1196-1202.

Boynton GM, Engel SA, Glover GH, Heeger DJ (1996) Linear systems analysis of functional magnetic resonance imaging in human V1. J Neurosci 16:4207-4221.

Brainard DH (1997) The psychophysics toolbox. Spat Vis 10:433-436.

Corbetta M, Shulman GL (2002) Control of goal-directed and stimulusdriven attention in the brain. Nat Rev Neurosci 3:201-215.

Corbetta M, Kincade JM, Ollinger JM, McAvoy MP, Shulman GL (2000) 
Voluntary orienting is dissociated from target detection in human posterior parietal cortex. Nat Neurosci 3:292-297.

Corbetta M, Kincade MJ, Lewis C, Snyder AZ, Sapir A (2005) Neural basis and recovery of spatial attention deficits in spatial neglect. Nat Neurosci 8:1603-1610.

Corbetta M, Patel G, Shulman GL (2008) The reorienting system of the human brain: from environment to theory of mind. Neuron 58:306-324.

Coull JT, Nobre AC (1998) Where and when to pay attention: the neural systems for directing attention to spatial locations and to time intervals as revealed by both PET and fMRI. J Neurosci 18:7426-7435.

Coull JT, Frith CD, Büchel C, Nobre AC (2000) Orienting attention in time: behavioural and neuroanatomical distinction between exogenous and endogenous shifts. Neuropsychologia 38:808-819.

de Fockert J, Rees G, Frith CD, Lavie N (2004) Neural correlates of attentional capture in visual search. J Cogn Neurosci 16:751-759.

Dimond SJ (1979) Performance by split-brain humans on lateralized vigilance tasks. Cortex 15:43-50.

Downar J, Crawley AP, Mikulis DJ, Davis KD (2000) A multimodal cortical network for the detection of changes in the sensory environment. Nat Neurosci 3:277-283.

Downar J, Crawley AP, Mikulis DJ, Davis KD (2001) The effect of task relevance on the cortical response to changes in visual and auditory stimuli: an event-related fMRI study. Neuroimage 14:1256-1267.

Downing PE, Jiang Y, Shuman M, Kanwisher N (2001) A cortical area selective for visual processing of the human body. Science 293:2470-2473.

Duncan J (1980) The locus of interference in the perception of simultaneous stimuli. Psychol Rev 87:272-300.

Forman SD, Cohen JD, Fitzgerald M, Eddy WF, Mintun MA, Noll DC (1995) Improved assessment of significant activation in functional magnetic resonance imaging (fMRI): use of a cluster-size threshold. Magn Reson Med 33:636-647.

Foucher JR, Otzenberger H, Gounot D (2004) Where arousal meets attention: a simultaneous fMRI and EEG recording study. Neuroimage 22:688-697.

Friedrich FJ, Egly R, Rafal RD, Beck D (1998) Spatial attention deficits in humans: A comparison of superior parietal and temporal-parietal junction lesions. Neuropsychology 12:193-207.

Gitelman DR, Nobre AC, Parrish TB, LaBar KS, Kim YH, Meyer JR, Mesulam M (1999) A large-scale distributed network for covert spatial attention: further anatomical delineation based on stringent behavioural and cognitive controls. Brain 122:1093-1106.

Hagler DJ Jr, Sereno MI (2006) Spatial maps in frontal and prefrontal cortex. Neuroimage 29:567-577.

Hagler DJ Jr, Riecke L, Sereno MI (2007) Parietal and superior frontal visuospatial maps activated by pointing and saccades. Neuroimage 35:1562-1577.

Hampshire A, Duncan J, Owen AM (2007) Selective tuning of the blood oxygenation level-dependent response during simple target detection dissociates human frontoparietal subregions. J Neurosci 27:6219-6223.

He BJ, Snyder AZ, Vincent JL, Epstein A, Shulman GL, Corbetta M (2007) Breakdown of functional connectivity in frontoparietal networks underlies behavioral deficits in spatial neglect. Neuron 53:905-918.

Heilman KM, Van Den Abell T (1980) Right hemisphere dominance for attention: the mechanism underlying hemispheric asymmetries of inattention (neglect). Neurology 30:327-330.

Heilman KM, Schwartz HD, Watson RT (1978) Hypoarousal in patients with the neglect syndrome and emotional indefference. Neurology 28:229-232.

Heilman KM, Watson RT, Valenstein E (1985) Neglect and related disorders. In: Clinical neuropsychology (Heilman KM, Valenstein E, eds), pp 243-293. New York: Oxford.

Hopfinger JB, Buonocore MH, Mangun GR (2000) The neural mechanisms of top-down attentional control. Nat Neurosci 3:284-291.

Husain M, Kennard C (1996) Visual neglect associated with frontal lobe infarction. J Neurol 243:652-657.

Husain M, Rorden C (2003) Non-spatially lateralized mechanisms in hemispatial neglect. Nat Rev Neurosci 4:26-36.

Indovina I, Macaluso E (2007) Dissociation of stimulus relevance and saliency factors during shifts of visuospatial attention. Cereb Cortex 17:1701-1711.

Jack AI, Patel GH, Astafiev SV, Snyder AZ, Akbudak E, Shulman GL, Corbetta M
(2007) Changing human visual field organization from early visual to extra-occipital cortex. PLoS One 2:e452.

Karnath HO, Ferber S, Himmelbach M (2001) Spatial awareness is a function of the temporal not the posterior parietal lobe. Nature 411:950-953.

Karnath HO, Fruhmann Berger M, Küker W, Rorden C (2004) The anatomy of spatial neglect based on voxelwise statistical analysis: a study of 140 patients. Cereb Cortex 14:1164-1172.

Kastner S, Ungerleider LG (2000) Mechanisms of visual attention in the human cortex. Annu Rev Neurosci 23:315-341.

Kastner S, Pinsk MA, De Weerd P, Desimone R, Ungerleider LG (1999) Increased activity in human visual cortex during directed attention in the absence of visual stimulation. Neuron 22:751-761.

Kelley TA, Serences JT, Giesbrecht B, Yantis S (2008) Cortical mechanisms for shifting and holding visuospatial attention. Cereb Cortex 18:114-125.

Kerr DL, Gusnard DA, Snyder AZ, Raichle ME (2004) Effect of practice on reading performance and brain function. Neuroreport 15:607-610.

Kincade JM, Abrams RA, Astafiev SV, Shulman GL, Corbetta M (2005) An event-related functional magnetic resonance imaging study of voluntary and stimulus-driven orienting of attention. J Neurosci 25:4593-4604.

Lancaster JL, Glass TG, Landkipalli BR, Downs H, Mayberg H, Fox PT (1995) A modality-independent approach to spatial normalization of tomographic images of the human brain. Hum Brain Mapp 3:209-223.

Liégeois F, Connelly A, Salmond CH, Gadian DG, Vargha-Khadem F, Baldeweg T (2002) A direct test for lateralization of language activation using fMRI: comparison with invasive assessments in children with epilepsy. Neuroimage 17:1861-1867.

Macaluso E, Patria F (2007) Spatial re-orienting of visual attention along the horizontal or the vertical axis. Exp Brain Res 180:23-34.

Macaluso E, Frith CD, Driver J (2002) Supramodal effects of covert spatial orienting triggered by visual or tactile events. J Cogn Neurosci 14:389-401.

Marois R, Ivanoff J (2005) Capacity limits of information processing in the brain. Trends Cogn Sci 9:296-305.

Mesulam MM (1981) A cortical network for directed attention and unilateral neglect. Ann Neurol 10:309-325.

Mevorach C, Humphreys GW, Shalev L (2009) Reflexive and preparatory selection and suppression of salient information in the right and left posterior parietal cortex. J Cogn Neurosci 21:1204-1214.

Molenberghs P, Mesulam MM, Peeters R, Vandenberghe RR (2007) Remapping attentional priorities: differential contribution of superior parietal lobule and intraparietal sulcus. Cereb Cortex 17:2703-2712.

Molenberghs P, Gillebert CR, Peeters R, Vandenberghe R (2008) Convergence between lesion-symptom mapping and functional magnetic resonance imaging of spatially selective attention in the intact brain J Neurosci 28:3359-3373.

Mort DJ, Malhotra P, Mannan SK, Rorden C, Pambakian A, Kennard C, Husain M (2003) The anatomy of visual neglect. Brain 126:1986-1997.

Nobre AC, Sebestyen GN, Gitelman DR, Mesulam MM, Frackowiack RS, Frith CD (1997) Functional localization of the system for visuospatial attention using positron emission tomography. Brain 120:515-533.

Oke A, Keller R, Mefford I, Adams RN (1978) Lateralization of norepinephrine in human thalamus. Science 200:1411-1413.

Ollinger JM, Shulman GL, Corbetta M (2001) Separating processes within a trial in event-related functional MRI I. The Method. Neuroimage 13:210-217.

O'Shea J, Johansen-Berg H, Trief D, Göbel S, Rushworth MF (2007) Functionally specific reorganization in human premotor cortex. Neuron 54:479-490.

Pardo JV, Fox PT, Raichle ME (1991) Localization of a human system for sustained attention by positron emission tomography. Nature 349:61-64.

Peers PV, Ludwig CJ, Rorden C, Cusack R, Bonfiglioli C, Bundesen C, Driver J, Antoun N, Duncan J (2005) Attentional functions of parietal and frontal cortex. Cereb Cortex 15:1469-1484.

Pelli DG (1997) The VideoToolbox software for visual psychophysics: transforming numbers into movies. Spat Vis 10:437-442.

Posner MI, Boies SJ (1971) Components of attention. Psychol Rev 78:391-408.

Posner MI, Cohen Y (1984) Components of visual orienting. In: Attention and performance X (Bouma H, Bowhuis D, eds), pp 531-556. Hillsdale, NJ: Erlbaum.

Posner MI, Petersen SE (1990) The attention system of the human brain. Annu Rev Neurosci 13:25-42. 
Posner MI, Inhoff A, Friedrich FJ, Cohen A (1987) Isolating attentional systems: a cognitive-anatomical analysis. Psychobiology 15:107-121.

Ringman JM, Saver JL, Woolson RF, Clarke WR, Adams HP (2004) Frequency, risk factors, anatomy, and course of unilateral neglect in an acute stroke cohort. Neurology 63:468-474.

Robertson IH, Manly T, Beschin N, Daini R, Haeske-Dewick H, Hömberg V, Jehkonen M, Pizzamiglio G, Shiel A, Weber E (1997) Auditory sustained attention is a marker of unilateral spatial neglect. Neuropsychologia 35:1527-1532.

Robertson IH, Mattingley JB, Rorden C, Driver J (1998) Phasic alerting of neglect patients overcomes their spatial deficit in visual awareness. Nature 395:169-172.

Robinson RG (1979) Differential behavioral and biochemical effects of right and left hemispheric cerebral infarction in the rat. Science 205:707-710.

Robinson RG (1985) Lateralized behavior and neurochemical consequences of unilateral brain injury in rats. In: Cerebral lateralization in nonhuman species, pp 135-156. New York: Academic.

Robinson RG, Coyle JT (1980) The differential effect of right versus left hemispheric cerebral infarction on catecholamines and behavior in the rat. Brain Res 188:63-78.

Rowland DJ, Garbow JR, Laforest R, Snyder AZ (2005) Registration of [18F]FDG microPET and small-animal MRI. Nucl Med Biol 32:567-572.

Ruff CC, Blankenburg F, Bjoertomt O, Bestmann S, Weiskopf N, Driver J (2009) Hemispheric differences in frontal and parietal influences on human occipital cortex: direct confirmation with concurrent TMS-fMRI. J Cogn Neurosci 21:1146-1161.

Sack AT, Camprodon JA, Pascual-Leone A, Goebel R (2005) The dynamics of interhemispheric compensatory processes in mental imagery. Science 308:702-704.

Saygin AP, Sereno MI (2008) Retinotopy and attention in human occipital, temporal, parietal, and frontal cortex. Cereb Cortex 18:2158-2168.

Schluppeck D, Glimcher P, Heeger DJ (2005) Topographic organization for delayed saccades in human posterior parietal cortex. J Neurophysiol 94:1372-1384.

Serences JT, Yantis S (2007) Spatially selective representations of voluntary and stimulus-driven attentional priority in human occipital, parietal, and frontal cortex. Cereb Cortex 17:284-293.

Serences JT, Shomstein S, Leber AB, Golay X, Egeth HE, Yantis S (2005) Coordination of voluntary and stimulus-driven attentional control in human cortex. Psychol Sci 16:114-122.

Sereno MI, Pitzalis S, Martinez A (2001) Mapping of contralateral space in retinotopic coordinates by a parietal cortical area in humans. Science 294:1350-1354.

Shulman GL, Astafiev SV, Franke D, Pope DL, Snyder AZ, McAvoy MP, Corbetta M (2009) Interaction of stimulus-driven reorienting and expectation in ventral and dorsal frontoparietal and basal ganglia-cortical networks. J Neurosci 29:4392-4407.

Silver MA, Ress D, Heeger DJ (2005) Topographic maps of visual spatial attention in human parietal cortex. J Neurophysiol 94:1358-1371.

Siman-Tov T, Mendelsohn A, Schonberg T, Avidan G, Podlipsky I, Pessoa L, Gadoth N, Ungerleider LG, Hendler T (2007) Bihemispheric leftward bias in a visuospatial attention-related network. J Neurosci 27:1127111278.

Stevens MC, Calhoun VD, Kiehl KA (2005) Hemispheric differences in hemodynamics elicited by auditory oddball stimuli. Neuroimage 26:782-792.

Sturm W, Willmes K (2001) On the functional neuroanatomy of intrinsic and phasic alertness. Neuroimage 14:S76-S84.

Sturm W, de Simone A, Krause BJ, Specht K, Hesselmann V, Radermacher I, Herzog H, Tellmann L, Müller-Gärtner HW, Willmes K (1999) Functional anatomy of intrinsic alertness: evidence for a fronto-parietalthalamic-brainstem network in the right hemisphere. Neuropsychologia 37:797-805.

Swisher JD, Halko MA, Merabet LB, McMains SA, Somers DC (2007) Visual topography of human intraparietal sulcus. J Neurosci 27:5326-5337.

Sylvester CM, Shulman GL, Jack AI, Corbetta M (2007) Asymmetry of anticipatory activity in visual cortex predicts the locus of attention and perception. J Neurosci 27:14424-14433.

Talairach J, Tournoux P (1988) Co-planar stereotaxic atlas of the human brain. New York: Thieme Medical.

Vallar G, Perani D (1986) The anatomy of unilateral neglect after righthemispere stroke lesions. A clinical/CT-scan correlation study in man. Neuropsychologia 24:609-622.

Van Essen DC (2005) A population-average, landmark- and surface-based (PALS) atlas of human cerebral cortex. Neuroimage 28:635-662.

Wilkins AJ, Shallice T, McCarthy R (1987) Frontal lesions and sustained attention. Neuropsychologia 25:359-365.

Woldorff MG, Hazlett CJ, Fichtenholtz HM, Weissman DH, Dale AM, Song AW (2004) Functional parcellation of attentional control regions of the brain. J Cogn Neurosci 16:149-165.

Yokoyama K, Jennings R, Ackles P, Hood P, Boller F (1987) Lack of heart rate changes during an attention-demanding task after right hemisphere lesions. Neurology 37:624-630. 